\title{
DESASTRES ASSOCIADOS A MOVIMENTOS DE MASSA: UMA REVISÃO DE LITERATURA
}

\author{
DISASTER ASSOCIATED WITH MASS MOVEMENTS: A LITERATURE REVIEW
}

\author{
DESASTRES ASOCIADOS A MOVIMIENTOS DEL \\ TERRENO: UNA REVISIÓN DE LA LITERATURA \\ Eduardo Samuel Riffel - Universidade Federal do Rio Grande do \\ Sul - Porto Alegre - Rio Grande do Sul - Brasil \\ edriffel@gmail.com
}
Laurindo Antonio Guasselli - Universidade Federal do Rio Grande do Sul - Porto Alegre - Rio Grande do Sul - Brasil laurindo.guasselli@ufrgs.br
Luiz Antonio Bressani - Universidade Federal do Rio Grande do Sul - Porto Alegre - Rio Grande do Sul - Brasil bressani@ufrgs.br

\section{Resumo}

Os movimentos de massa constituem, juntamente com as enchentes, um dos desastres que apresenta 0 maior grau de recorrência em todo o mundo e, portanto, são causadores de danos e prejuízos às sociedades, particularmente em cidades densamente povoadas em zonas de relevo acidentado. No entanto, muitas vezes, ainda não existe um consenso acerca dos conceitos utilizados pelos diversos órgãos de pesquisa e de defesa civil. Assim, faz-se necessário organizar as informações referentes a esses eventos adversos através de análise, identificação e classificação. Como diversas medidas de mitigação e de auxílio devem ser tomadas pós-desastre, tais medidas precisam ser coerentemente estabelecidas. Diante disso, este trabalho objetiva revisar a literatura dos conceitos relacionados com desastres, tais como suscetibilidade, vulnerabilidade, perigo e risco, buscando conceitos atuais relacionados aos principais tipos de movimentos de massa e suas características.

Palavras-chave: suscetibilidade, vulnerabilidade, perigo, risco.

\section{Abstract}

The mass movements are, together with the flooding, one of the disasters with the highest degree of recurrence in the world and, therefore, are causing damages to companies, particularly in densely populated cities in hilly areas.However, often there is still no consensus among the concepts used by the various bodies of research and civil defense. Thus, it is necessary to organize information regarding adverse events, through his analysis, identification and classification. Different measures of mitigate and assistance should be taken post-disaster, such measures need to be coherently established. Therefore, this work aims to review the literature of the concepts related to disasters as susceptibility, vulnerability, hazard and risk, seeking current concepts related to the main types of mass movements and their characteristics.

Keywords: susceptibility, vulnerability, hazard, risk. 


\section{Resumen}

Los movimientos de masa, junto con las inundaciones, constituyen uno de los desastres que presenta mayor grado de incidencia y son, por eso, causadores de daños y prejuicios para las sociedades, particularmente en ciudades densamente pobladas en zonas montañosas. A pesar de eso, es frecuente verificar que aún no existe un acuerdo relacionado a los conceptos usados por los diferentes órganos de investigación y de defensa civil. Por lo tanto, es necesario organizar las informaciones relacionadas a esos desastres mediante análisis, identificación y clasificación. Como varias medidas de mitigación y auxilio deben ser tomadas después de cada desastre, esas medidas necesitan ser coherentemente establecidas. Partiendo de esa percepción, el objetivo de este trabajo es revisar la literatura de los conceptos relacionados a los desastres, tales como susceptibilidad, vulnerabilidad, peligro y riesgo, buscando conceptos actuales relacionados a los principales tipos de movimientos de masas y sus características.

Palabras-claves: susceptibilidad, vulnerabilidad, peligro, riesgo.

Introdução

O relatório apoiado pelas Nações Unidas revela que, em 2013, 22 milhões de pessoas em todo o mundo foram deslocadas em decorrência de desastres naturais provocados, em grande parte, por terremotos ou eventos climáticos. O relatório "Estimativas Globais 2014: pessoas deslocadas por desastres", realizado pelo Centro de Monitoramento de Deslocados Internos do Conselho Norueguês de Refugiados (CMDI), mostra que o risco de deslocamento devido a desastres mais do que dobrou nos últimos 40 anos, em grande parte devido ao crescimento e a concentração das populações urbanas, especialmente nos países mais vulneráveis (UN, 2014).

O aumento das ocorrências de desastres e das áreas de risco traz grandes preocupações sobre suas causas e consequências. No entanto, como esse cenário é recente, ainda não existe um consenso em relação aos conceitos utilizados pelos diversos órgãos de pesquisa e de defesa civil. Com o objetivo de organizar as informações referentes a esses eventos adversos, faz-se necessário o trabalho de análise, identificação e classificação desses eventos. Como diversas medidas de mitigação e de auxílio devem ser tomadas pós-desastre, tais medidas precisam ser coerentemente estabelecidas.

Os movimentos de massa constituem, juntamente com as enchentes, um dos desastres que apresenta o maior grau de recorrência em todo o mundo, sendo ambos causadores de danos e prejuízos às sociedades, particularmente em cidades densamente povoadas em zonas de relevo acidentado. Estimativas mostram que a quantidade de movimentos de massa registrados no mundo supera as de outras ameaças naturais, como 
sismos, furacões e vulcões (Chene Lee, 2004). Embora este tema seja amplamente estudado em diversos países, o Brasil ainda carece de materiais que reúnam esse conceitual. Diante disso, este trabalho tem por objetivo realizar uma revisão de literatura dos temas relacionados a movimentos de massa.

\section{Desastres}

Inicialmente, faz-se necessário conhecer um pouco do conceito de desastres, que podem ser conceituados, de forma simplificada, como o resultado do impacto de um fenômeno natural extremo ou intenso sobre um sistema social, causando sérios danos e prejuízos que excedem a capacidade dos afetados em conviver com o impacto (Tobine Montz, 1997; UNDP, 2004). De acordo com a Política Nacional da Defesa Civil, na Resolução $N^{\circ} 3$ de 02/07/99 (Brasil, 2012), desastre é o resultado de eventos adversos, naturais ou provocados pelo homem, sobre um ecossistema (vulnerável), causando danos humanos, materiais e/ou ambientais e consequentes prejuízos econômicos e sociais. A Defesa Civil, no Brasil, obedecendo às normativas da Política Nacional de Defesa Civil, classifica os desastres como naturais e humanos, sendo os desastres humanos aqueles gerados pelas ações ou omissões humanas, como incêndios industriais, contaminação de rios, entre outros. Os desastres naturais são causados pelo impacto de um fenômeno natural de grande intensidade sobre uma área ou região povoada, podendo ou não ser agravados pelas atividades antrópicas (Castro, 2003).

Segundo Oliveira (2010), desastre natural é todo fenômeno natural de grande intensidade, agravado ou não pela atividade humana. As diversas ocorrências de desastres naturais podem ser exemplificadas por inundações, abalos sísmicos, erosões, deslizamentos, incêndios florestais, tempestades, ciclones etc., todos passíveis de posicionamento e mensuração da extensão da área comprometida pelo desastre. De acordo com Scheurenet al. (2008), apenas no ano de 2007, 133 países foram atingidos por desastres naturais. Os prejuízos não se restringem apenas aos danos econômicos, contando-se também os ambientais e sociais. Para Braga et al. (2006), a grande incidência de eventos adversos justifica a necessidade de aprofundar o conhecimento científico sobre os efeitos desiguais destes 
eventos na população, assim como o desenvolvimento de metodologias de mensuração da vulnerabilidade a estes eventos.

\section{Desastres no Brasil}

Os desastres que ocorrem no Brasil são, na sua maioria, de origem atmosférica. A precipitação, seja em excesso ou em escassez, é o elemento atmosférico que mais contribui para a ocorrência de desastres na região Sul do Brasil. Os desastres possuem características e magnitudes variadas. Em consequência da adequação que o homem faz as suas necessidades, o ambiente, muitas vezes, não comporta todas essas modificações espaciais, acarretando diversos impactos ambientais (Teixeira e Satyamurty, 2004).Segundo a Instrução Normativa $\mathrm{N}^{\circ}$ 01, de 24 de Agosto de 2012 do Ministério das Cidades, desastre é o resultado de eventos adversos, naturais ou provocados pelo homem, sobre um cenário vulnerável, causando grave perturbação ao funcionamento de uma comunidade ou sociedade, envolvendo extensivas perdas e danos humanos, materiais, econômicos ou ambientais, que excedem a sua capacidade de lidar com o problema usando meios próprios. No Brasil, as pesquisas sobre movimentos de massa ganham grande impulso com o trabalho de Guidicinie Nieble (1984), que apresenta uma sistemática de classificação de movimentos de massa, seus agentes e causas, além de métodos para cálculo de estabilidade de taludes. Os desastres, no Brasil, segundo Furlan et al. (2011), são resultado de uma combinação da ocorrência de eventos adversos com a vulnerabilidade física e/ou socioeconômica, destacando-se, portanto, a necessidade de conhecer espacialmente quais áreas são suscetíveis à ocorrência de eventos adversos e quais possuem características potencializadoras do risco.

Os conceitos de desastres têm sido amplamente discutidos por diversos autores nos últimos anos e, baseado no referencial analisado nesse trabalho, constata-se um consenso de que desastres consistem na ocorrência de um evento adverso que afeta um sistema social. Percebe-se que, para um evento ser considerado desastre, é preciso considerar o sistema social como elemento atingido. Para discutirmos os temas relacionados a movimentos de massa, devemos conhecer alguns conceitos que serão brevemente descritos a seguir, sendo eles: Suscetibilidade e Vulnerabilidade, Perigo e Risco. 


\section{Suscetibilidade e Vulnerabilidade}

Segundo Spink (2014), nas geociências, há dois fatores a serem considerados na análise de movimentos de massa: a suscetibilidade (maior ou menor propensão de instabilidade do solo, decorrente de características geológicas e geomórficas, somadas a valores de precipitação altos), e a vulnerabilidade (predisposição de pessoas e/ou construções a serem afetadas por ocasião de um desastre). A vulnerabilidade está obviamente associada ao uso e a ocupação do solo.

O IPT (2007) indica a potencialidade de ocorrência de processos naturais e induzidos em uma dada área, expressando-se segundo classes de probabilidade de ocorrência, relacionando suscetibilidade com processos naturais, e vulnerabilidade com processos induzidos.

Saito (2004) define suscetibilidade como uma característica inerente ao meio, representando a fragilidade do ambiente. Já Reckziegel (2012) define a avaliação da suscetibilidade como uma característica inerente ao meio, que representa a fragilidade do ambiente em relação aos movimentos de massa, e é analisada através dos fatores de predisposição para a ocorrência.

Baseado nos itens acima, pode-se concluir que a suscetibilidade está ligada apenas ao sistema natural, sem levar em consideração a ação antrópica, representando somente a fragilidade do ambiente, diferentemente do conceito de vulnerabilidade que, segundo o Ministério das Cidades (2012), é definido como o grau de perda para um dado elemento, grupo ou comunidade dentro de uma determinada área passível de ser afetada por um fenômeno ou processo. Para Silva (2012, p.1), vulnerabilidade é "o grau de resistência de um sistema socioeconômico em relação ao impacto dos perigos naturais e desastres tecnológicos ou ambientais.” É determinada pelo grau de consciência da população, por sua condição de vida, pela infraestrutura e pelas políticas públicas relacionadas à gestão de desastres. Para Galderisi et al. (2010), vulnerabilidade seria a combinação do estado de um sistema com outros fatores, como a capacidade de enfrentar e se recuperar de um evento catastrófico.

De maneira geral, percebe-se uma concordância pela maior parte dos autores em relação aos conceitos de suscetibilidade e vulnerabilidade, salvo algumas exceções em que a suscetibilidade foi relacionada com o componente socioeconômico. Para se trabalhar com vulnerabilidade em 
uma comunidade, por exemplo, deve-se levar em consideração as condições humanas, visto que são elas que mais influenciam na severidade de um desastre. Fica evidente, dessa forma, que a vulnerabilidade está ligada à condição humana e sua capacidade de enfrentar um evento adverso.

\section{Perigo e Risco}

O termo perigo (hazard) é, muitas vezes, confundido com o termo risco (risk), sendo que a tradução livre desses conceitos do inglês para o português pode ser a causa disso. No presente artigo, serão associados os termos com seus conceitos originais, isto é, perigo - hazard e risco - risk.

Para Bateira (2006), sempre houve muita confusão na utilização dos termos risco (risk) e perigo (hazard). Segundo Smith (1992; 1996), a discussão sobre as diferenças entre 'risk' e 'hazard' parece mais evidente. Ao definir risco como a probabilidade de ocorrência de um 'hazard', o autor aborda de forma direta o problema da distinção entre os dois termos. Assim, um 'hazard' não é mais do que uma ameaça potencial para a humanidade. Esta ameaça poderá ser de importância diversa consoante o tipo de consequências que acarreta, quer para o homem, quer para o ambiente. Dessa forma, para este autor, a magnitude e a intensidade das ocorrências que influem direta ou indiretamente na vida humana é informação essencial para a definição. Segundo Castro (2003, p.80), perigo "implica a existência do homem, que estima o que é, e o que não é um dano", visto que nem sempre os fenômenos naturais causam prejuízos às comunidades, podendo, então, ser considerado apenas como um evento. Nesse sentido, Castro apresenta a definição adotada pelas Nações Unidas, a qual aponta que o perigo natural é "a probabilidade de ocorrência, em um determinado período, em uma determinada área, de um fenômeno natural potencialmente danoso" (NAÇÕES UNIDAS,1984, apud Castro, 2003, p. 80). Assim, pode-se entender basicamente que perigo consiste em uma situação potencial para provocar danos, sendo que, associando o conceito aos movimentos de massa, essa situação potencial pode ser provocada por um fenômeno natural.

De acordo com Montoya (2013), embora o conceito de risco tenha ganhado muita importância nos trabalhos técnicos em anos recentes, existem diferentes percepções e definições do que é risco. O termo risco indica a probabilidade de ocorrência de algum dano a uma população 
(pessoas ou bens materiais). É uma condição potencial de ocorrência de um acidente (Nogueira, 2002).

A Resolução n ${ }^{\circ} .2$ de 12/12/94 (DOU, 1995) e a Resolução no .03 de 02/07/99 (DOU, 1999) definem risco como um acontecimento futuro, como a medida de danos ou prejuízos potenciais ao homem, expressa em termos de probabilidade.

O risco indica se um determinado evento, com uma intensidade específica, seja ele de origem natural ou humana, é mais ou menos provável, e quais os danos e prejuízos esperar, sempre levando em consideração os pontos fracos e fortes de uma comunidade em relação ao evento adverso. Quanto maior a probabilidade de o evento ocorrer com grande intensidade, e quanto mais significativos os danos e prejuízos previsíveis nestes casos, maior o risco (DEFESA CIVIL-SC, 2005).

Risco é também compreendido como a relação entre a possibilidade de ocorrência de um dado processo ou fenômeno e a magnitude de danos ou consequências sociais e/ou econômicas sobre um dado elemento, grupo ou comunidade. Quanto maior a vulnerabilidade, maior o risco. Para o JTC-1 (2008), risco é uma medida de probabilidade e severidade de um efeito adverso à saúde, à propriedade ou ao meio ambiente. Assim, o risco é frequentemente estimado pelo produto da probabilidade versus consequências. No entanto, uma interpretação mais geral de risco envolve uma comparação da probabilidade das consequências não em formato de produto.

Serpa (2001) define risco como a medida de perda econômica, de danos à vida humana e/ou de impactos ambientais, resultante da combinação entre a frequência de ocorrência e a magnitude das perdas ou danos (consequências). Segundo Farah (2003), no Brasil, há uma carência absoluta de dados confiáveis no que diz respeito ao tipo de risco. Quando se trata de risco, deve-se considerar a suscetibilidade à ocorrência de fenômenos e à vulnerabilidade (fragilidade social, densidade demográfica, infraestrutura, conhecimento e percepção do risco, situação econômica etc.) do sistema que está sob análise. Buscando sintetizar os conceitos de Suscetibilidade, Vulnerabilidade, Perigo e Risco, segue abaixo um quadro com os principais autores tratados nessa pesquisa e a sua definição em linhas gerais de cada conceito. 
Percebe-se, a partir do Quadro 1, que ocorre um consenso por parte dos autores pesquisados nesse artigo em relação aos conceitos de suscetibilidade e vulnerabilidade, sendo a primeira considerada como uma propensão do terreno à ocorrência de fenômeno e/ou processo físico, e a segunda como a capacidade da sociedade enfrentar o desastre.

Também há consenso no conceito de perigo. No entanto, alguns autores citam outros dois termos. A ameaça, para algumas referências do Quadro 1, é considerada sinônimoao vocábulo perigo, bem como perigosidade,muito utilizado por autores portugueses, como descrito por Bateira (2006).O risco é utilizado a partir de duas abordagens, uma que o considera como uma probabilidade, que pode ser calculado e estimado, e que representa a maioria das referências citadas nesse artigo, outra que considera que o risco não pode ser quantificado, que ele deve ser analisado de forma empírica e isolada, sendo a segunda representativada minoria dos autores citados.

Quadro 1- Conceitos de suscetibilidade, vulnerabilidade, perigo e risco elaborados pelas fontes citadas nesse trabalho.

\begin{tabular}{|c|c|c|c|}
\hline & Conceito: & Fontes: & Descrição: \\
\hline 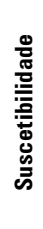 & $\begin{array}{l}\text { Suscetibilidade } \\
\text { é a propensão } \\
\text { do terreno à } \\
\text { ocorrência de } \\
\text { fenômeno e/ou } \\
\text { processo físico }\end{array}$ & $\begin{array}{l}\text { Saito, 2004; Kobiyama, 2006; IPT, 2007; } \\
\text { Fell et al., 2008; Julião et al., 2009; } \\
\text { Galderisi et al., 2010; Sobreira e Souza, } \\
\text { 2012; Diniz, 2012; Reckziegel, 2012; } \\
\text { Silva, 2012; Coutinho, 2013; MINISTÉRIO } \\
\text { DAS CIDADES, 2013; Riffel e Guasselli, } \\
\text { 2013; }\end{array}$ & $\begin{array}{l}\text { Os autores consideram suscetibilidade } \\
\text { como a propensão maior ou menor } \\
\text { de ocorrência de um fenômeno, com } \\
\text { baseapenas nas características físicas. }\end{array}$ \\
\hline 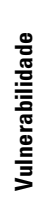 & $\begin{array}{l}\text { Vulnerabilidade } \\
\text { é a capacidade } \\
\text { da sociedade } \\
\text { de enfrentar e/ } \\
\text { ou suportar } \\
\text { um evento } \\
\text { catastrófico }\end{array}$ & $\begin{array}{l}\text { Saito, 2004; Kobiyama, 2006; IPT, 2007; } \\
\text { Fell et al., 2008; Julião et al., 2009; } \\
\text { Galderisi et al., 2010; Reckziegel, 2012; } \\
\text { Silva, 2012; Spink, } 2014 .\end{array}$ & $\begin{array}{l}\text { Alguns autores abordam vulnerabilidade } \\
\text { considerando as características } \\
\text { socioeconômicas. Alguns também } \\
\text { trazem o conceito de resiliência, que } \\
\text { consiste na capacidade da sociedade de } \\
\text { suportar efeitos adversos. }\end{array}$ \\
\hline \multirow{2}{*}{ } & $\begin{array}{l}\text { Perigo é uma } \\
\text { situação potencial } \\
\text { para provocar } \\
\text { danos }\end{array}$ & $\begin{array}{c}\text { Smith, 1992; 0jeda, 1997; Bateira, 2006; } \\
\text { Kobiyama, 2006; Fell et al., 2008; Julião } \\
\text { et al., 2009; Hermelin, 2007; Cardona et } \\
\text { al., 2010. }\end{array}$ & $\begin{array}{l}\text { Alguns autores utilizam o termo ameaça } \\
\text { e perigosidade, como sinônimos de } \\
\text { perigo, consistindo numa potencialidade, } \\
\text { e não probabilidade como é classificado } \\
\text { o risco. Segundo os autores, o perigo não } \\
\text { pode ser estimado. }\end{array}$ \\
\hline & $\begin{array}{l}\text { Ameaça é uma } \\
\text { situação potencial } \\
\text { para provocar } \\
\text { danos }\end{array}$ & $\begin{array}{l}\text { CAMPOS, 1999; LAVELL, } \\
\text { 1999;CARDONA, } 2001 .\end{array}$ & $\begin{array}{l}\text { Utilizam o conceito de ameaça ao invés } \\
\text { do conceito de perigo, considerados } \\
\text { por alguns autores como sinônimos } \\
\text { (Campos, 1999; UN/ISDR, 2009; } \\
\text { Monteiro \& Pinheiro, 2012). }\end{array}$ \\
\hline
\end{tabular}




\begin{tabular}{|c|c|c|c|}
\hline & Conceito: & Fontes: & Descrição: \\
\hline \multirow{3}{*}{ 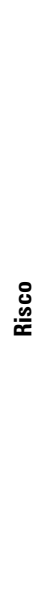 } & $\begin{array}{c}\text { Risco é a } \\
\text { probabilidade de } \\
\text { ocorrência de } \\
\text { evento danoso } \\
\text { a partir da } \\
\text { relação entre } \\
\text { Suscetibilidade e } \\
\text { Perigo. }\end{array}$ & $\begin{array}{l}\text { Cerri e Amaral, 1998; Aneas de Castro, } \\
\text { 2000; Nogueira, 2002; Castro, 2003; } \\
\text { Bateira, 2006; Carvalho e Galvão, 2006; } \\
\text { Kobiyama, 2006; Carvalho, Macedo e } \\
\text { Ogura, 2007; Fell et al., 2008; JTC-1, } \\
\text { 2008; Julião et al., 2009. }\end{array}$ & $\begin{array}{l}\text { Consideram risco uma probabilidade } \\
\text { que pode ser estimada e calculada. 0s } \\
\text { termos mais utilizados para a formatação } \\
\text { do conceito são suscetibilidade e perigo. }\end{array}$ \\
\hline & $\begin{array}{l}\text { Risco é } \\
\text { caracterizado } \\
\text { pela interação } \\
\text { entre ameaça e } \\
\text { vulnerabilidade. }\end{array}$ & $\begin{array}{l}\text { Campos, 1999; Lavell, 1999; Cardona, } \\
2001 .\end{array}$ & $\begin{array}{l}\text { Utilizam o conceito de ameaça para } \\
\text { trabalhar com o risco, visto que alguns } \\
\text { autores consideram ameaça como } \\
\text { sinônimo de perigo (Campos, 1999; UN/ } \\
\text { ISDR, 2009; Monteiro \& Pinheiro, 2012). }\end{array}$ \\
\hline & & $\begin{array}{c}\text { Otway, 1992; Duclos, 2002; Lagadec e } \\
\text { Guilhou, 2004; Granjo, } 2004 .\end{array}$ & $\begin{array}{l}\text { Consideram o risco associado aos } \\
\text { termos ameaça e vulnerabilidade, no } \\
\text { entanto não consideram que o risco } \\
\text { seja uma probabilidade, e não pode ser } \\
\text { estimado. }\end{array}$ \\
\hline
\end{tabular}

Org: os autores

\section{Movimentos de Massa}

Aqui serão abordados os principais tipos de movimentos de massa e os de maior ocorrência no Brasil, que são os deslizamentos e as corridas. Tais termos correspondem a processos definidos pelo IPT (Instituto de Pesquisas Tecnológicas), propostos por Augusto Filho (1992), que é uma das classificações mais utilizadas atualmente. Devido aos diversos tipos de movimentos de massa, há uma grande dificuldade em se identificar corretamente qual o tipo de movimento ocorreu em um determinado evento. Em alguns casos, estes se confundem, dificultando ainda mais o entendimento dos mecanismos que influenciam na sua deflagração. Por exemplo, os deslizamentos e as corridas que, muitas vezes, encontram-se associados, pois o início da corrida está vinculado à ocorrência de um deslizamento anterior (Guidicinie Nieble, 1984; IPT, 1991; Selby, 1993).

Segundo Silveira (2008), na bibliografia internacional, os movimentos de solo e rocha são conhecidos e classificados como landslides pelos autores americanos, landslips pelos ingleses, massmovements por alguns engenheiros geotécnicos e geomorfólogos, slope movements pela maioria dos engenheiros geotécnicos e masswasting pela maioria dos geomorfólogos. 
De maneira geral, pode-se definir landslides como um termo utilizado para denominar diversos tipos de movimentos de massa, quedas, tombamentos, deslizamentos, fluxos e/ou afundamentos ao longo de placas.

Analisando os diferentes conceitos internacionais para os movimentos em vertentes ou encostas, Nummer (2003) conclui que o termo landslides pode ser utilizado como sinônimo de movimento de massa (massmovements). Isso significa, em termos mais amplos, todo e qualquer movimento de materiais terrosos ou rochosos, não importando sua forma, sua velocidade e nem o processo que o gerou.

\section{Deslizamentos}

Os deslizamentos são processos marcantes na evolução das encostas, caracterizando-se por movimentos rápidos (m/h a m/s), com limites laterais e profundidade bem definidos. Os volumes instabilizados podem ser facilmente identificados ou, pelo menos, inferidos. Podem envolver solo, saprólito, rocha e depósitos (Figura 1). São subdivididos em função do mecanismo de ruptura, geometria e material que mobilizam.

\section{Figura 1 - Deslizamento planar}

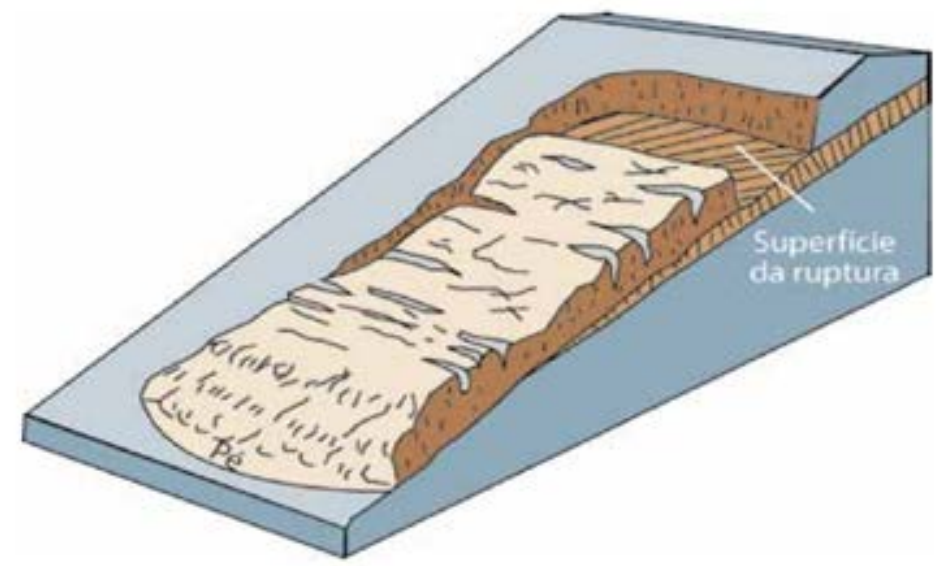

Fonte: Highland, 2008

Os principais agentes deflagradores deste processo são as chuvas. Os índices pluviométricos críticos variam de acordo com a região, sendo 
menores para os deslizamentos induzidos e maiores para os generalizados (IPT, 2007). Entretanto, deve-se considerar que outros autores consideram que a força gravitacional é mais importante na ocorrência de deslizamentos do que a chuva. (Christofoletti, 1980; Giannini e Riccomini, 2001).

Para a Secretaria Nacional de Defesa Civil (2003), deslizamento é um fenômeno provocado pelo escorregamento de materiais sólidos, como solos, rochas, vegetação e/ou material de construção ao longo de terrenos inclinados, denominados de "encostas", "pendentes" ou "escarpas".

Existem vários tipos de deslizamentos propriamente ditos, como os planares ou translacionais, os circulares ou rotacionais, os em cunha e os induzidos. A geometria destes movimentos varia em função da existência ou não de estruturas ou planos de fraqueza nos materiais movimentados que condicionam a formação das superfícies de ruptura.

Os deslizamentos planares ou translacionais em solo são processos muito frequentes na dinâmica das encostas serranas brasileiras, ocorrendo predominantemente em solos pouco desenvolvidos das vertentes com altas declividades. Os deslizamentos induzidos ou causados pela ação antrópica são aqueles cuja deflagração é causada pela execução de cortes e aterros inadequados, pela concentração de águas pluviais e servidas, pela retirada da vegetação etc. Muitas vezes, estes deslizamentos induzidos mobilizam materiais produzidos pela própria ocupação, envolvendo massas de solo de dimensões variadas, lixo e entulho (IPT, 2015).

\section{Corridas}

As corridas de detritos são movimentos gravitacionais complexos de massa, ligados a eventos pluviométricos excepcionais. Ocorrem apartir de deslizamentos nas encostas e mobilizam grandes volumes de material, sendo o seu escoamento ao longo de um ou mais canais de drenagem, tendo comportamento líquido viscoso e alto poder de transporte (IPT, 2007).

Estes fenômenos são mais raros que os deslizamentos, porém podem provocar consequências de magnitudes superiores devido ao seu grande poder destrutivo e extenso raio de alcance, mesmo em áreas planas (Figura 2).

As corridas de detritos abrangem uma gama variada de denominações na literatura nacional e na internacional (corrida de lama -mudflow, corrida de massa - massflow, corrida de blocos - debrisflow etc.), 
principalmente em função de suas velocidades e das características dos materiais que mobilizam.

Figura 2 - Corridas de detritos

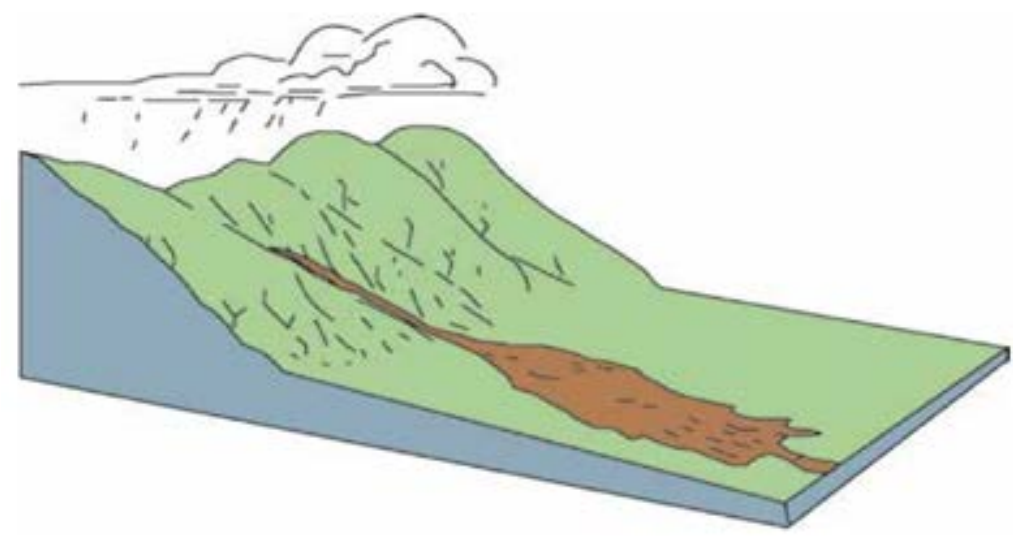

Fonte: Highland, 2008

Segundo Stiny (1910), fluxo de detritos é a descrição de uma torrente escoando em uma montanha carregando sólidos suspensos e transportando determinada quantidade de massa erodida. Como a quantidade de massa transportada aumenta, em determinado momento, ela se transforma em uma massa viscosa contendo a mistura de água, solo, areia, pedras, rochas e madeira, de modo que este escoamento se assemelha à lava vulcânica transitando em um vale. O fluxo de detritos é um mecanismo de ruptura complexo que pode se manifestar em decorrência do aumento de poro pressões nas descontinuidades da rocha provocado por chuvas intensas e prolongadas, ou ainda pela ação de sismos. Os movimentos definidos como fluxos diferenciam-se dos demais movimentos de massa devido à características peculiares, tais como: velocidade elevada, alta capacidade de erosão e destruição e transporte de detritos a grandes distâncias em períodos de tempo muito curtos (Kanjiet al., 1997; Massad et al., 1997 e 1998).

As corridas de detritos são consideradas o tipo de movimento de massa de maior poder destrutivo e de maiorcarregamento de materiais. Segundo Cruden e Varnes (1996), o termo fluxo de detritos ou debrisflow representa um fenômeno completo de movimento de massa 
(escorregamento e propagação de material grosseiro), o qual inclui o início do deslocamento em uma declividade acentuada, escoando rapidamente em um canal confinado à deposição em um leque de detritos.

Quadro 2 - Conceitos de deslizamentos e corridas elaborados

pelas fontes citadas nesse trabalho.

\begin{tabular}{|c|c|c|c|}
\hline & Conceito & Fontes & Descrição \\
\hline 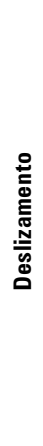 & $\begin{array}{l}\text { Fenômeno provocado } \\
\text { pelo escorregamento de } \\
\text { materiais sólidos como } \\
\text { solos, rochas, vegetação e/ } \\
\text { ou material de construção } \\
\text { ao longo de terrenos } \\
\text { inclinados. }\end{array}$ & $\begin{array}{l}\text { Christofoletti, 1980; Augusto Filho, } \\
\text { 1992; Pinheiro et al., 2000; Giannini e } \\
\text { Riccomini, 2001; Avelar et al., 2002; } \\
\text { Nogueira, 2002; Secretaria Nacional } \\
\text { de Defesa Civil, 2003; Nummer et al., } \\
\text { 2003; Herrmann, 2004; Marcelino, } \\
\text { 2004; Saito, 2004; Znamensky, } \\
\text { 2005; Bateira, 2006; G0MES, 2006; } \\
\text { VANACOR, 2006; Monguilhott, 2008; } \\
\text { Silveira, 2008; Tominaga, 2009; } \\
\text { Montoya, 2013. }\end{array}$ & $\begin{array}{l}\text { Em linhas gerais, os autores } \\
\text { revisados concordam com o conceito } \\
\text { de deslizamento. Alguns autores, } \\
\text { porém, divergem em relação à causa } \\
\text { principal da ocorrência dos eventos, } \\
\text { em alguns casos relacionados à } \\
\text { força gravitacional (Christofoletti, } \\
\text { 1980; Giannini e Riccomini, 2001), } \\
\text { em outros à precipitação (IPT, 2007). } \\
\text { A maioria dos autores, no entanto, } \\
\text { sustenta que diversas variáveis } \\
\text { devem ser levadas em consideração, } \\
\text { tais como geomorfologia, geologia, } \\
\text { solos, precipitação etc. }\end{array}$ \\
\hline 窇 & $\begin{array}{l}\text { Movimentos gravitacionais } \\
\text { complexos de massa ligados } \\
\text { a eventos pluviométricos } \\
\text { excepcionais. Ocorrem a } \\
\text { partir de deslizamentos } \\
\text { nas encostas e mobilizam } \\
\text { grandes volumes de } \\
\text { material. } 0 \text { escoamento } \\
\text { ocorre ao longo de um ou } \\
\text { mais canais de drenagem. } \\
\text { Têm comportamento líquido } \\
\text { viscoso e alto poder de } \\
\text { transporte. }\end{array}$ & $\begin{array}{l}\text { Stiny, 1910; Christofoletti, 1980; } \\
\text { Augusto Filho, 1992; Cruden e } \\
\text { Varnes, 1996; Kanji et al., 1997; } \\
\text { Massad et al., } 1997 \text { e 1998; Pinheiro } \\
\text { et al., 2000; Avelar et al., 2002; } \\
\text { Nummer et al., 2003; Saito, 2004; } \\
\text { Marcelino, 2004; Znamensky, } \\
\text { 2005; Bateira, 2006; Gomes, 2006; } \\
\text { Vanacor, 2006; Highland, 2008; } \\
\text { Monguilhott, 2008; Silveira, 2008; } \\
\text { Gramani, 2013; Kobiyama e Michel, } \\
\text { 2015. }\end{array}$ & $\begin{array}{l}\text { A maioria dos autores concorda na } \\
\text { conceituação de corridas. Variam } \\
\text { apenas na nomenclatura utilizada, } \\
\text { tais como fluxos, fluxos de detritos, } \\
\text { fluxos de massa, corridas de } \\
\text { massa. A respeito desse conceito } \\
\text { destacamos o trabalho “Bibliografia } \\
\text { dos trabalhos de fluxos de detritos } \\
\text { ocorridos no Brasil no período } \\
\text { de 1949-2014", de Kobiyama e } \\
\text { Michel (2015), onde consta a quase } \\
\text { totalidade dos trabalhos envolvendo } \\
\text { a temática de corridas no Brasil. }\end{array}$ \\
\hline
\end{tabular}

Org: os autores

De maneira geral, percebe-se uma concordância por parte dos autores revisados quanto à conceituação de deslizamentos, exceto pela sua gênese. Alguns autores consideram, como principal causador, a força gravitacional, enquanto outros consideram a precipitação como o deflagrador principal dos deslizamentos.

Quanto às corridas, os autores citados abordam o conceito da mesma forma, variando apenas algumas terminologias, tais como: corridas, fluxos, corridas de massa etc. Também concordam que tanto para os 
deslizamentos quanto para as corridas devem ser levados em consideração diversos elementos (geologia, solos, precipitação, geomorfologia etc.).

Com base nos conceitos analisados, percebe-se que os desastres associados a movimentos de massa ocorrem pela conjunção de diversos fatores, como os deslizamentos e/ou as corridas em áreas vulneráveis e de risco. Destaca-se que os movimentos de massa por si só não geram desastres, a menos que causem impactos na sociedade.

\section{Considerações Finais}

O aumento da ocorrência de desastres no Brasil representa também um aumento de perdas e danos com relação direta na perda de vidas e nos impactos na economia de estados e municípios. Por isso, é necessário mais investimentos na prevenção a partir de uma correta análise das áreas e das causas dos desastres. É importante ressaltar que a legislação nacional avançou em relação à redução de desastres, comona Lei № 12.608 de 2012, que institui a política nacional de Proteção Civil e define como responsabilidade da União, dos Estados, do Distrito Federal e dos Municípios a adoção de medidas necessárias à redução dos riscos de desastre. Essa lei abrange também as ações de prevenção, mitigação, preparação, resposta e recuperação voltadas à proteção e à defesa civil.

É importante também ressaltar os compromissos que os governos em nível mundial têm tomado em relação à diminuição das consequências dos desastres diante da importância que as discussões sobre esses eventos adversos têm alcançado. O Marco de Sendai para a Redução de Riscos de Desastres 2015-2030 representa um importante passo para uma redução significativa das mortes, destruições e deslocamentos causados pelos desastres. Os 187 países que compareceram à Terceira Conferência Mundial da ONU para a Redução de Riscos de Desastres também reafirmaram a necessidade de antecipar, planejar e reduzir o risco para proteger pessoas, comunidades e países de forma mais efetiva. Construir uma maior resiliência também é considerada uma medida “urgente e crítica” (ONU, 2015).

Segundo Bateira (2006), a análise dos riscos naturais aparece cada vez mais como uma análise que integra o meio físico e a ação humana. O homem é um elemento da vida e da dinâmica terrestre, o que torna mais difícil a análise do risco natural separadamente da análise de todos os outros riscos, nomeadamente os riscos tecnológicos. A relação do homem 
com o meio tem se intensificado, mas apenas atualmente a sociedade está percebendo as consequências de suas ações. A análise dos conceitos de risco e vulnerabilidade, conceitos ligados diretamente à sociedade, são conceitos essenciais ao se trabalhar com movimentos de massa.

De forma objetiva, a partir das referências analisadas, pode-se considerar a suscetibilidade como uma característica física/natural do meio para a ocorrência de um evento. Essas características podem tornar determinada porção do relevo suscetível a deslizamento, independente da influência antrópica. O perigo é, por sua vez, uma situação potencial de ocorrência de um determinado evento, contanto que alguns autores utilizem o termo "ameaça" para referir esse conceito. A vulnerabilidade é um conceito relacionado ao ser humano e a sua capacidade de suportar o desastre. Risco ainda é o conceito que gera mais confusão, e a maioria dos autores, levando em consideração o perigo e a vulnerabilidade, classifica o risco como a probabilidade de ocorrência de um desastre, considerando também que o risco é um dado que pode ser quantificado.

Em relação aos movimentos de massa, percebe-se um consenso em relação à diferenciação entre deslizamentos e corridas. São claras as características entre as suas dimensões e seu poder destrutivo, sendo relevante discutir alguns fatores que podem levar à sua gênese, como a influência da força gravitacional e da água, por exemplo. Ao analisarmos os desastres a partir dos movimentos de massa, devemos considerar uma conjunção de fatores que são os elementos naturais e sociais do meio, representados pela suscetibilidade, perigo, vulnerabilidade e risco. Entretanto, esses conceitos ainda devem ser muito debatidos, pois os conhecimentos teóricos sobre os desastres e movimentos de massa podem contribuir para o entendimento dos processos envolvidos e o avanço das pesquisas.

\section{Referências}

ANEAS DE CASTRO, S. D. Riesgos y peligros: una visión desde lá Geografía. Revista Electrónica de Geografía y Ciencias Sociales. Barcelona, n. 60, 15 de ma 2000.

AUGUSTO FILHO, O. Caracterização geológico-geotécnica voltada à estabilização de encostas: uma proposta metodológica. In: CONFERÊNCIA BRASILEIRA SOBRE ESTABILIDADE DE ENCOSTAS, 1, 1992, Rio de Janeiro. Anais... Rio de Janeiro: ABMS/ABGE. 1992. p. 721-733. 
AVELAR, A.S.; LACERDA, W.A.; COELHO NETTO, A.L. Análise de susceptibilidade a movimentos de massa no Maciço da Tijuca (RJ) utilizando SIG. In: CONFERÊNCIA BRASILEIRA SOBRE ESTABILIDADE DE ENCOSTAs, 3, 2002, Rio de Janeiro. Anais... Rio de Janeiro: ABMS/ABGE. 2002. p.133-140.

BATEIRA, C. Recuperação de paisagens de terraços e prevenção de riscos naturais. Porto-Portugal. TERRISC: (SUDOE III B). 2006.

BRAGA, T. M; et al. Avaliação de metodologias de mensuração de risco e vulnerabilidade social a desastres naturais associados à mudança climática. In: ENCONTRO NACIONAL DE ESTUDOS POPULACIONAIS, 15,. 2006, Caxambu, Anais eletrônicos... ABEP, 2006. Disponível em: <www.abep.nepo.unicamp.br/ encontro2006/.../_204.pdf> Acesso em: 15 jul. 2014.

BRASIL. Instrução Normativa $\mathrm{N}^{\circ}$ 01, de 24 de Agosto de 2012. Diário Oficial da União, Poder Executivo, Brasília: DF, 31 de ago. 2012 p. 30-39.

. Mapeamento de Riscos em Encostas e Margem de Rios.Celso Santos Carvalho, Eduardo Soares de Macedo e Agostinho Tadashi Ogura, organizadores - Brasília: Ministério das Cidades; Instituto de Pesquisas Tecnológicas - IPT, 2007. $176 \mathrm{p}$.

. Anuário Brasileiro de Desastres Naturais 2012. Brasília: Ministério da Integração Nacional, 2012.

. Manual de Desastres. Vol. 1 - Desastres, Secretaria Nacional de Defesa Civil 2003. 69. Disponível em: http://www.defesacivil.mg.gov.br/conteudo/ arquivos/manuais/Manuais-de- Defesa-Civil/Manual-PLANEJAMENTO-1.pdf> Acesso em 23 mar 2015.

BUENO, L. S.; LAPOLLI, E. M.; LAPOLLI, F. R. Estudo em Áreas de Ocupação Urbana com Fatores de Risco: O Caso do Bairro Córrego Grande - Florianópolis SC. In: Congreso Interamericano de Ingenieria Sanitaria y Ambiental, 28,. 2002, Cancun, Mexico, Anais... 2002.

CARDONA, O.D., BERTONI, J.C., GIBBS, T., HERMELIN, M., Y LAVELL, A. Entendimiento y gestión del riesgo asociado a las amenazas naturales: un enfoque científico integral para América Latina y El Caribe. In: ICSU - LAC. Ciencia para una vida mejor: desarrollando programas científicos regionales en areas prioritarias para América Latina y El Caribe. Volumen2. ICSU - LAC / CONACYT: Rio de Janeiro y Ciudad de México, 2010, 88p.

CASSETI, V. Ambiente e apropriação do relevo. São Paulo: Contexto, 1991. 147p.

- Geomorfologia. [S.l.]: Notas explicativas [2005]. Disponível em: $<$ http://www.funape.org.br/geomorfologia/>. Acesso em: 22 nov. 2014.

CASTRO, A. L. C. Manual de desastres: desastres naturais. Brasília: MIN, 2003. 174p.

CASTRO, J. F. M. Mapa Morfodinâmico: Uma Abordagem Metodológica de Uso de Sistema de Informação Geográfica. Revista Geociências, UNESP, Rio Claro, v. 17, p.161-185, 2003.

CHEN C.Y. LEE W. C. Damages to school infrastructure and development to disaster prevention education strategy after Typhoon Morakot in Taiwan, 
Disaster Prevention and Management: An International Journal, v. 21 n. 5, p.541555, 2004.

COUTINHO, R. Q. (Coord. e Org.). Parâmetros para a cartografia geotécnica e diretrizes para medidas de intervenção de áreas sujeitas a desastres naturais. Recife: Ministério das Cidades; Universidade Federal de Pernambuco; GEGEP; DECivil, 2013. 376p. (Documento Técnico)

CHRISTOFOLETTI, A. Geomorfologia. São Paulo: Edgard Blucher, 1980. 188 p.

DEFESA CIVIL-SC, Os CONSEGS e a redução de riscos. Governo do Estado de Santa Catarina, $2^{\mathrm{a}}$ ed. 2008. 154p. Disponível em: http://www.defesacivil.pr.gov. br/arquivos/File/publicacoes/LivroCONSEGsReducaodeRiscos.pdf $>$. Acesso em: 23 mar 2016.

DINIZ, N. C. Cartografia geotécnica por classificação de unidades de terreno e avaliação de suscetibilidade e aptidão. Revista Brasileira de Geologia de Engenharia e Ambiental, v. 2, n. 1, p. 29-77, 2012.

DUCLOS, D. (2002), Société-monde: letempsdesruptures, Paris, La Découverte. 2002, $256 \mathrm{p}$.

FARAH, F. Ocupação urbana e instabilidade de encostas. Coleção Habitare habitação e encostas. São Paulo: IPT, v. 2, 2003. 312p.

FELL, R. et al. Guidelines for landslide susceptibility, hazard and risk zoning for land-use planning. Engineering Geology, v. 102, p. 83-111, 2008.

FERREIRA, D. Sistema de informações geográficas participativo (SIG-P) na prevenção de desastres ambientais: estudo de caso do morro do baú em Ilhota/ SC. 132 f. Dissertação ((Mestrado Profissional em Planejamento Territorial e Desenvolvimento Socioambiental) -Universidade do Estado de Santa Catarina,Mestrado Profissional em Planejamento Territorial e Desenvolvimento Socioambiental, Florianópolis, 2012.

FURLAN, M. C.; LACRUZ, M. S. P.; SAUSEN, T. M. Vulnerabilidade socioeconômica à ocorrência de eventos extremos: proposta metodológica. In: SIMPÓSIO BRASILEIRO DE SENSORIAMENTO REMOTO, 15,. Curitiba. Anais... São José dos Campos: INPE, 2011. p. 4540-4546, Disponível em: <http:// urlib.net/3ERPFQRTRW/3A3P672>. Acesso em: 15 mar 2015.

GALDERISI, A., CEUDECH, A., FERRARA, F. F., PROFICE, A. S.Integration of different vulnerabilities vs. Natural and Na-tech Hazards, 2010 apud Tapsell, S; McCarthy, S; Faulkner, H \& Alexander, M (2010): Social Vulnerability and Natural Hazards. CapHaz-Net WP4 Report, Flood Hazard Research Centre FHRC, Middlesex University, London.

GIANNINI, P.C.F.; RICCOMINI, C. Sedimentos e processos sedimentares. In: TEIXEIRA, W. et al. Decifrando a Terra. São Paulo: Of. de Textos, p. 167-190, 2001.

GRAMANI, M., A corrida de detritos no Ribeirão Cagado, Serra do Mar, Municipio de Cubatao-SP. In VI Conferencia Brasileira de Encostas. Angra dos Reis 2013.

GRANJO, P. Quando o conceito de risco se torna perigoso. Análise Social, v. 41, n. 181, 1167-1179, Imprensa de Ciências Sociais 2006. 
GOMES, R. A. T. Modelagem de Previsão de Movimentos de Massa a Partir da Combinação de Modelos de Escorregamentos e Corridas de Massa. 2006. $101 \mathrm{f}$. Tese (Doutorado em Geografia) Universidade Federal de Goiás, 2006.

GUIDICINI, G. \& NIEBLE, C. M. Estabilidade de Taludes Naturais e de Escavação. São Paulo: 2a ed. Edgard Blücher, 1984. 194p.

HERRMANN, M. L.; PELLERIN, J. R. G. M.; SAITO, S. M. Análise das ocorrências de escorregamentos no estado de Santa Catarina com base nos formulários de avaliação de danos da defesa civil 1980 a 2003. In: Simposio Brasileiro de Desastres Naturais, 1. Anaiss... Florianópolis: GEDN/UFSC, 2004.

HERMELÍN, H. Sistemas morfogénicos contrastados em el Norte de la Cordillera Central Colombiana. Revista de la Academia Colombiana de Ciencias. v. 31, n. 119, p. 199-216, 2007.

INSTITUTO DE PESQUISAS TECNOLÓGICAS DO ESTADO DE SÃO PAUL.; Curso de treinamento de técnicos municipais para o mapeamento e gerenciamento de áreas urbanas com risco de escorregamentos e inundações. Relatório Técnico 74186-205. São Paulo. 2004.

INSTITUTO DE PESQUISAS TECNOLÓGICAS DO ESTADO DE SÃO PAULO; Mapeamento e diagnóstico das áreas de risco associado a processos de instabilização do terreno nas encostas dos Morros de Arujá, SP. Relatório Técnico 77889-205. São Paulo, 2005.

INSTITUTO DE PESQUISAS TECNOLÓGICAS DO ESTADO DE SÃO PAULO; Mapeamento de áreas de alto e muito alto risco a deslizamentos e inundações do município de Potim, SP. Relatório Técnico 142750-205. São Paulo, 2015.

JOINT TECHNICAL COMMITTEE ON LANDSLIDES AND ENGINEERED SLOPES - JTC1 - FELL, R. COROMINAS, J. BONNARD, C. CASCINI, L. LEROI, E. SAVAGE, W. (Org.) Engineering Geology, 2008.

JULIÃO, R. P. (Coord.) et al. Guia metodológico para a produção de cartografia municipal de risco e para a criação de Sistemas de Informação Geográfica (SIG) de base municipal. Lisboa: Autoridade Nacional de Protecção Civil; DirecçãoGeral do Ordenamento do Território e Desenvolvimento Urbano; Instituto Geográfico Português, 2009.

KANJI, M. A., CRUZ, P. T., MASSAD, F., Debris flow affecting the Cubatão Oil Refinery, Brazil, Landslides, v.5, p. 71-82, 2008.

KOBIYAMA, M.; MICHEL, G.P. Histórico de ocorrência de fluxos de detritos e seus estudos no Brasil. In: Encontro Nacional de Engenharia de Sedimentos, 11, João Pessoa/PB, Anais..., 2014. 20p.

KOBYAMA, M. Prevenção de Desastres Naturais: Conceitos Básicos. Curitiba: Ed. Organic Trading , 2006. 109 p.

LAGADEC, P. e GUILHOU, X. O Fim do Risco Zero, Lisboa, Instituto Piaget. (2004).

MARCELINO, E. V. Mapeamento de áreas suscetíveis a escorregamento no município de Caraguatatuba (SP) usando técnicas de sensoriamento remoto e 
SIG. São José dos Campos. 2004. 228p. Dissertação (Mestrado em Sensoriamento Remoto) - Inpe, 2004.

MINISTÉRIO DAS CIDADES. Capacitação em mapeamento e gerenciamento de risco. [S.l.,]. Disponível em: http://www.defesacivil.mg.gov.br/conteudo/ arquivos/manuais/Mapeamento.pdf. Acesso em: 1 novembro 2015.

MONGUILHOTT, M. Estudo de áreas suscetíveis a movimentos de massa na rodovia RS/486 - Rota do Sol. Porto Alegre: UFRGS, 2008. 115 f. Dissertação (Mestrado em Sensoriamento Remoto) - Universidade Federal do Rio Grande do Sul.

MONTEIRO, J. B. \& PINHEIRO, D. R. C. O desastre natural como fenômeno induzido pela sociedade: abordagens teóricas e metodologias operacionais para identificação/mitigação de desastres naturais. Revista Geografia, Juiz de Fora, v.2, n.1, p.1-9, 2012.

MONTOYA, C.A.H. Incertezas, vulnerabilidade e avaliação de risco devido a deslizamento em estrada. 2013, 250 f. Tese de Doutorado em Geotecnia / UnB, 2013.

NOGUEIRA, F.R. Políticas públicas municipais para gerenciamento de riscos ambientais associados a escorregamentos em áreas de ocupação subnormal. 2002, 256 f. Tese (Doutorado em Geociências e Meio Ambiente) - Universidade Estadual Paulista - UNESP. Rio Claro. 256p. 2002.

NUMMER, V.A Parâmetros Geológico-Geotécnicos controladores dos movimentos de massa na rota do sol/ RS 486 - Itati, RS. 2003. Tese (Doutorado) Universidade Federal do Rio Grande do Sul, Porto Alegre, 2003.

OJEDA, A.O. Crecidas e inundaciones como riesgo hidrológico Un planteamiento didáctico. Revista Lurralde: Investigación de Espácio, v. 20, p. 261-283, 1997.

OLIVEIRA G.G. - Modelos para Previsão, espacialização e análise das áreas inundáveis na bacia hidrográfica do Rio Caí, RS. 2010, 149 f. Dissertação (Mestrado em Sensoriamento Remoto) Universidade Federal do Rio Grande do Sul, Porto Alegre, 2010.

OTWAY, H. (1992), Public wisdom, expert fallibility: toward a contextual theory of risk, In S. Krimsky e D. Golding (eds.), Social Theories of Risk, Westport, Praeger, 1992. p. 215- -228.

PINHEIRO, R.J.B.; BRESSANI, L.A.; BICA, A.V.D. Movimentos de massa associados à encosta da Serra Geral no Estado do Rio Grande do Sul. In: GEOSUL, 2000, Porto Alegre. GEOSUL. Porto Alegre: ABMS, Anais, v.1. p.115-127, 2000.

RECKZIEGEL, E. W.;Identificação e mapeamento das áreas com perigo de movimento de massa no município de Porto Alegre, RS. 2012. 90 f. Dissertação (Mestrado), Universidade Federal do Rio Grande do Sul, Porto Alegre, 2012.

RIFFEL, E.S.; GUASSELLI, L. A. Mapeamento de áreas suscetíveis a movimentos de massa na bacia hidrográfica do rio Paranhana, RS. In: Laurindo AntonioGuasselli; Guilherme Garcia de Oliveira; Rita de Cássia Marques Alves. (Org.). EVENTOS EXTREMOS NO RIO GRANDE DO SUL: INUNDAÇÕES E 
MOVIMENTOS DE MASSA. 1ed.Porto Alegre: Editora Evangraf, v. 1, p. 119138, 2013.

SAITO, S. Estudo analítico da suscetibilidade a escorregamentos e quedas de blocos no maciço central de Florianópolis - SC. 2004. 133 f. Dissertação (Mestrado de Geografia). Departamento de Geociências da Universidade Federal de Santa Catarina, Florianópolis-SC, 2004, 133p.

SCHEUREN, J.M; POLAIN, O.; BELOW, R.; GUHA-SAPIR, D.; PONSERRE, S. 2008. Annual Disaster Statistical Review - The Numbers and Trends 2007. CRED - Centre for Research on the Epidemiology of Disasters. 2008, Université Catholique de Louvain, Brussels, Belgium. Disponível em: http://www.emdat. be/Documents. Acesso em: 1 nov. 2014.

SELBY, M. Earth's changing surface: an introduction to geomorphology. New York: Oxford University Press, 1985.

SERPA, R. R. As metodologias de análises de risco e seu papel no licenciamento de industrias e atividades perigosas. In: Freitas, C.M. Souza, M.F. Machado, J.M. (org) Acidentes industriais ampliados: desafios e perspectivas para o controle e a prevenção. Rio de Janeiro: Editora FIOCRUZ, 2001.

SILVA, F. A. Percepção dos Riscos e Desastres Ambientais dos Anos de 2008 e 2011 em Jaraguá Do Sul - SC. 2012. 106 f. Dissertação (Mestrado Em Geografia). Universidade Federal De Mato Grosso Do Sul. Três Lagoas, 2012.

SILVEIRA, R. M. Comportamento Geotécnico de um Solo Coluvionar de São Vendelino (RS). 2008. 302 f. Tese (Doutorado em Engenharia Civil). Universidade Federal do Rio Grande do Sul. Porto Alegre, 2008.

SMITH, K. Environmental hazards: assessing risk \& reducing disaster. London: Routledge, 1992. 324p.

SOBREIRA, F. G.; SOUZA, L. A. de. Cartografia geotécnica aplicada ao planejamento urbano. Revista Brasileira de Geologia de Engenharia e Ambiental, v. 2, n. 1, p. 79-97, 2012.

SPINK, M. J.;Produção de sentidos no cotidiano: uma abordagem teóricometodológica para análise das práticas discursivas. In: SPINK. (Org.). Práticas discursivas e produção de sentidos no cotidiano: aproximações teóricas e metodológicas. Rio de Janeiro: Centro Edelstein de Pesquisas Sociais (Edição virtual), 2014.

STINY, J. Die Muren. Verlag der Wagner'schenUniversitätsbuchhandlung, Innsbruck, 1910. Debris Flows. (English translation by M. Jakob and N. Skermer, 1997, 106pp.), EBA Engineering Consultants, Vancouver, Canada.

TEIXEIRA, M.S.; SATYAMURTY, P. Dynamical and synoptic characteristics of heavy rainfall episodes in southern Brazil. Monthly Weather Review, v. 135, p. 598-617, 2007.

TOBIN, G. A; MONTZ, B. E.Natural Hazards: explanation and investigation. New York: The Guilford Press, 1997. 388p.

TOMINAGA, L. K; SANTORO, J.; AMARAL, R. (organizadores). Desastres Naturais: Conhecer para prevenir. São Paulo: Instituto Geológico, 2009. 
UN ISDR, 2014. Global Assessment Report on Disaster Risk Reduction: Revealing Risk, Redefining. Disponível em: http://www.preventionweb.net/english/hyogo / gar/2014/en/home/index.html Acesso em: 15 dez. 2014.

UN/ISDR. Terminología sobre Reducción del Riesgo de Desastres. Ginebra, Suiza: $\mathrm{UN}, 2009$

UNDP - United Nations Development Programme. Reducing disaster risk: a challenge for development. New York, USA: UNDP, 2004, 129p.

VANACÔR, R.N. Sensoriamento remoto e geoprocessamento aplicados ao mapeamento das áreas susceptíveis a movimentos de massa na região nordeste do Estado do Rio Grande do Sul. 2006, 132p. 2006. 132 f. Dissertação (Mestrado em Sensoriamento Remoto). Universidade Federal do Rio Grande do Sul

ZNAMENSKY, D. Mecanismos de formação das corridas detríticas em ambientes tropicais úmidos e a pesquisa de estruturas para o controle e manejo desses fenômenos. 2005. Tese (Doutorado) Universidade de São Paulo, 2005.

Eduardo Samuel Riffel - Possui Graduação em Geografia pela Universidade Federal de Santa Maria e Mestrado pela Universidade Federal do Rio Grande do Sul. Atualmente é Doutorando em Geografia pela Universidade Federal do Rio Grande do Sul e Técnico em Geoprocessamento na $1^{\text {a }}$ Divisão de Levantamento subordinada à Diretoria do Serviço Geográfico.

Laurindo Antonio Guasselli - Possui Graduação em Geografia pela Universidade Federal do Rio Grande do Sul. Mestrado em Sensoriamento Remoto e Doutorado em Recursos Hídricos e Saneamento Ambiental pela mesma Universidade. Atualmente é Professor do Departamento de Geografia pela Universidade Federal do Rio Grand

Luiz Antonio Bressani - Possui Graduação em Engenharia Civil pela Universidade Federal do Rio Grande do Sul. Mestrado em Engenharia Civil pela mesma Universidade. Possui Doutorado em Mecânica dos Solos pelo Imperial College de Londres. Atualmente Professor Titular do Departamento Engenharia Civil da Universidade Federal do Rio Grande do Sul.

Recebido para publicação em 02 de maio de 2016 Aceito para publicação em 19 de maio de 2016 